G. Nakamura

Nagoya Math. J.

Vol. 151 (1998), 129-143

\title{
THE EXISTENCE OF SYMMETRIC RIEMANN SURFACES DETERMINED BY CYCLIC GROUPS
}

\author{
GOU NAKAMURA
}

\begin{abstract}
Let $n>1, m \geq 1, g \geq 3$ and $\gamma$ be given integers. The purpose of this paper is to determine the relations of $n, m, g$ and $\gamma$ for the existence of the symmetric Riemann surfaces $S$ of type $(n, m)$ with genus $g$ and species $\gamma$. If $n$ is an odd prime, the relations are known in [3]. In the case that $n$ is odd, we shall show the analogous result when $E(S)$ is isomorphic to a cyclic group $\mathbf{Z}_{2 n}$ and when the quotient space $S / E(S)$ is orientable.
\end{abstract}

\section{$\S 1$. Introduction}

Let $S$ be a compact Riemann surface. We denote by $E(S)$ the group of analytic homeomorphisms and anti-analytic homeomorphisms of $S$ onto itself and by $A(S)$ its subgroup of analytic homeomorphisms. If $A(S)$ is isomorphic to a cyclic group $\mathbf{Z}_{n}$ of order $n$ and the quotient space $S / A(S)$ is of genus $m$, then $S$ is called a Riemann surface of type $(n, m)$. An element $T$ in $E(S) \backslash A(S)$ is called a symmetry on $S$ if $T^{2}(=T \circ T)=I_{S}$ (the identity map). A compact Riemann surface with symmetries is said to be symmetric. For a symmetry $T$ on $S$ the quotient space $S /\langle T\rangle$ is a Klein surface. Let $k$ be the number of boundary components of $S /\langle T\rangle$. Then we define the species $\operatorname{sp}(T)$ of $T$ by

$$
\operatorname{sp}(T)= \begin{cases}k & (\text { if } S /\langle T\rangle \text { is orientable }) \\ -k & (\text { if } S /\langle T\rangle \text { is non-orientable }) .\end{cases}
$$

In this paper we suppose that $E(S)$ is isomorphic to a cyclic group $\mathbf{Z}_{2 n}$ of order $2 n$. Then for such a symmetric Riemann surface $S$, the symmetry $T$ on $S$ is uniquely determined. Hence we define the species of $S$ by that of $T$.

Let $n>1, m \geq 1, g \geq 3$ and $\gamma$ be given integers. The purpose of this paper is to determine the relations of $n, m, g$ and $\gamma$ for the existence of the

Received June 19, 1996.

Revised May 22, 1997. 
symmetric Riemann surfaces $S$ of type $(n, m)$ with genus $g$ and species $\gamma$. If $n$ is an odd prime, the relations are known in [3]. In the case that $n$ is odd, we shall show the analogous result when $E(S)$ is isomorphic to a cyclic group $\mathbf{Z}_{2 n}$ and when the quotient space $S / E(S)$ is orientable.

\section{$\S 2$. Non-Euclidean crystallographic groups}

Let $H=\{z \in \mathbf{C} \mid \Im z>0\}$ be the upper half plane. With each matrix $A=\left(\begin{array}{ll}a & b \\ c & d\end{array}\right)$ with $a, b, c, d \in \mathbf{R}$ and with $\operatorname{det} A= \pm 1$, we associate the mapping

$$
f_{A}: H \rightarrow H ; z \mapsto \begin{cases}\frac{a z+b}{c z+d} & \text { if } \operatorname{det} A=1, \\ \frac{a \bar{z}+b}{c \bar{z}+d} & \text { if } \operatorname{det} A=-1 .\end{cases}
$$

Then $E(H)=\left\{f_{A} \mid \operatorname{det} A= \pm 1\right\}$ and $A(H)=\left\{f_{A} \mid \operatorname{det} A=1\right\}$. We regard $E(H)$ as a topological space by means of the inclusion $E(H) \hookrightarrow P G L(2, \mathbf{R})$. A discrete subgroup $\Gamma$ of $E(H)$ is called a non-Euclidean crystallographic group (shortly an NEC group) if the quotient $H / \Gamma$ is compact. An NEC group $\Gamma$ is called a Fuchsian group if $\Gamma \subset A(H)$, and a proper NEC group otherwise. For a proper NEC group $\Gamma, \Gamma^{+}=\Gamma \cap A(H)$ is called the canonical Fuchsian group of $\Gamma$.

In general, each NEC group $\Gamma$ is formed by the generators

$$
\begin{array}{llll}
x_{i} & \in \Gamma^{+} & ; & i=1, \cdots, r \\
e_{i} & \in \Gamma^{+} & ; & i=1, \cdots, k, \\
c_{i j} & \in \Gamma \backslash \Gamma^{+} & ; & i=1, \cdots, k, \quad j=0, \cdots s_{i}, \\
a_{i}, b_{i} & \in \Gamma^{+} & ; & i=1, \cdots, g \text { if } H / \Gamma \text { is orientable, } \\
d_{i} & \in \Gamma \backslash \Gamma^{+} & ; & i=i, \cdots, g \text { if } H / \Gamma \text { is non-orientable, }
\end{array}
$$

satisfying the relations

$$
\begin{array}{ll}
x_{i}^{m_{i}}=I_{H} & ; \quad i=1, \cdots, r, \\
e_{i}^{-1} c_{i 0} e_{i} c_{i s_{i}}=I_{H} & ; \quad i=1, \cdots, k, \\
c_{i, j-1}^{2}=c_{i j}^{2}=\left(c_{i, j-1} c_{i j}\right)^{n_{i j}}=I_{H} & ; \quad i=1, \cdots, k, j=1, \cdots, s_{i}, \\
x_{1} \cdots x_{r} e_{1} \cdots e_{k}\left[a_{1}, b_{1}\right] \cdots\left[a_{g}, b_{g}\right]=I_{H} & \text { if } H / \Gamma \text { is orientable }, \\
x_{1} \cdots x_{r} e_{1} \cdots e_{k} d_{1}^{2} \cdots d_{g}^{2}=I_{H} & \text { if } H / \Gamma \text { is non-orientable }
\end{array}
$$

where $\left[a_{i}, b_{i}\right]=a_{i} b_{i} a_{i}^{-1} b_{i}^{-1}$. We call $x_{i}$ an elliptic element, $c_{i j}$ a reflection of $\Gamma$. Then the signature $\sigma(\Gamma)$ of $\Gamma$ is written by

(1) $\sigma(\Gamma)=\left(g ; \pm ;\left[m_{1}, \cdots, m_{r}\right] ;\left\{\left(n_{11}, \cdots, n_{1 s_{1}}\right), \cdots,\left(n_{k 1}, \cdots, n_{k s_{k}}\right)\right\}\right)$, 
where "+" means that $H / \Gamma$ is orientable, and "-" means that $H / \Gamma$ is non-orientable. This "+" or "-" is called the sign of $\sigma(\Gamma)$ and denoted by $\operatorname{sign}(\sigma(\Gamma))$. We call $g$ the genus, $m_{i}$ the proper periods, $n_{i j}$ the periods, and $\left(n_{i 1}, \cdots, n_{i s_{i}}\right)$ the period-cycles of $\sigma(\Gamma)$. If there are no proper periods, we write [-] in place of $\left[m_{1}, \cdots, m_{r}\right]$. If there are no periods in the period-cycle, we write $(-)$ in place of $\left(n_{i 1}, n_{i 2}, \cdots, n_{i s_{i}}\right)$. If there are no period-cycles, we write $\{-\}$ in place of $\left\{\left(n_{11}, \cdots, n_{1 s_{1}}\right), \cdots,\left(n_{k 1}, \cdots, n_{k s_{k}}\right)\right\}$.

For an NEC group $\Gamma$ with signature (1), the Gauss-Bonnet theorem shows that the non-Euclidean area $\mu(F)$ of a fundamental region $F$ of $\Gamma$ is given by

$$
\mu(F)=2 \pi\left(\alpha g+k-2+\sum_{i=1}^{r}\left(1-\frac{1}{m_{i}}\right)+\frac{1}{2} \sum_{i=1}^{k} \sum_{j=1}^{s_{i}}\left(1-\frac{1}{n_{i j}}\right)\right),
$$

where $\alpha=2$ if $\operatorname{sign}(\sigma(\Gamma))=$ "+", $\alpha=1$ if $\operatorname{sign}(\sigma(\Gamma))=$ "-". This does not depend on the choice of fundamental regions. We define the area of $\sigma(\Gamma)$ by $\mu(F) / 2 \pi$ and denote it by $\mu(\Gamma)$.

Let $\Gamma^{\prime}$ be an NEC group and $\Gamma$ a subgroup of $\Gamma^{\prime}$ with finite index. Then $\Gamma$ is an NEC group, and the following formula (called the Riemann-Hurwitz relation) is fulfilled:

$$
\frac{\mu(\Gamma)}{\mu\left(\Gamma^{\prime}\right)}=\left[\Gamma^{\prime}: \Gamma\right]
$$

\section{$\S 3$. The main result}

Let $m_{1}, m_{2}, \cdots, m_{k}$ be integers. We denote the least common multiple of $\left\{m_{1}, m_{2}, \cdots, m_{k}\right\}$ by l.c.m. $\left\{m_{1}, m_{2}, \cdots, m_{k}\right\}$.

THEOREM 1. Let $n>1$ be an odd integer and $m \geq 1, g \geq 3$ and $\gamma$ integers. Then there exists a symmetric Riemann surface $S$ of type $(n, m)$ with genus $g(S)=g, s p(S)=\gamma, E(S) \cong \mathbf{Z}_{2 n}$ and with the orientable quotient $S / E(S)$ if and only if:

There exist non-negative integers $r, t$ and divisors $d_{1}, \cdots, d_{r+t}(\neq 1)$ of $n$ and an integer $k \geq 1$ such that:

(a) If $m=1$, then $r \geq 2$. If $m=2$, then $r \geq 1$.

(b) $g=n\left(m-1+\sum_{i=1}^{r}\left(1-\frac{1}{d_{i}}\right)\right)+1$. 
(c) $m+1-k$ is even and non-negative.

(d) $0 \leq t \leq k$.

(e) $\gamma=n\left(k-\sum_{i=1}^{t}\left(1-\frac{1}{d_{r+i}}\right)\right)(\geq 0)$.

(f) If $r+t>0$, then 1.c.m. $\left\{d_{1}, \cdots, d_{r+t}\right\}=$ l.c.m. $\left\{d_{1}, \cdots, d_{i-1}, d_{i+1}, \cdots\right.$, $\left.d_{r+t}\right\}$ for every $i$.

(g) If $k=m+1$, then 1.c.m. $\left\{d_{1}, \cdots, d_{r+t}\right\}=n$.

We note that the divisors $d_{1}, \cdots, d_{r+t}$ are not necessarily distinct.

If $n$ is an odd prime $p$, our theorem is reduced to the following

Corollary 1. [3; Theorem 2.1] There exists a symmetric Riemann surface $S$ of type $(p, m)$ with $g(S)=g, s p(S)=\gamma, E(S) \cong \mathbf{Z}_{2 p}$ and with the orientable quotient $S / E(S)$ if and only if:

There exist non-negative integers $r, t$ and an integer $k \geq 1$ such that:

(a) If $m=1$, then $r \geq 2$. If $m=2$, then $r \geq 1$.

(b) $g=p(r+m-1)-r+1$.

(c) $m+1-k$ is even and non-negative.

(d) $0 \leq t \leq k$.

(e) $\gamma=p(k-t)+t$.

(f) If $r+t>0$, then $r+t \geq 2$.

(g) If $k=m+1$, then $r+t \neq 0$.

\section{$\S 4$. The proof of our theorem}

We shall use the following lemma (see [4; Lemma 3.1.1]).

LEMMA 1. Let $m_{1}, m_{2}, \cdots, m_{k}>0$ be odd integers and $N$ a (positive) multiple of $M=$ l.c.m. $\left\{m_{1}, m_{2}, \cdots, m_{k}\right\}$. Then the following conditions are equivalent to each other.

(1) There exist $\xi_{1}, \cdots, \xi_{k}$ in $\mathbf{Z}_{N}$ such that $o\left(\xi_{i}\right)=m_{i}$ and $\xi_{1}+\cdots+\xi_{k}=0$ in $\mathbf{Z}_{N}$. 
(2) For every i, l.c.m. $\left\{m_{1}, \cdots, m_{i-1}, m_{i+1}, \cdots, m_{k}\right\}=M$.

Proof of our theorem. First we shall show the "only if" part. By our assumption $g \geq 3, H$ is the universal covering surface for $S$, so that there exists a torsion-free Fuchsian group $\Gamma_{S}$ satisfying $S \cong H / \Gamma_{S}$. Then the signature of $\Gamma_{S}$ is $\sigma\left(\Gamma_{S}\right)=(g ;+;[-] ;\{-\})$. We denote by $N_{S}$ the normalizer of $\Gamma_{S}$ in $E(H)$. We shall show that the signatures of $N_{S}$ and $N_{S}^{+}\left(=N_{S} \cap\right.$ $A(H))$ have the following forms with some non-negative integers $r, k(1 \leq$ $k \leq m+1)$ and divisors $d_{1}, \cdots, d_{r}$ of $n$ :

$$
\begin{aligned}
\sigma\left(N_{S}\right) & =\left(\frac{m+1-k}{2} ;+;\left[d_{1}, d_{2}, \cdots, d_{r}\right] ;\{\overbrace{(-), \cdots,(-)}^{k}\}\right), \\
\sigma\left(N_{S}^{+}\right) & =\left(m ;+;\left[d_{1}, d_{1}, d_{2}, d_{2}, \cdots, d_{r}, d_{r}\right] ;\{-\}\right) .
\end{aligned}
$$

We note that $d_{1}, \cdots, d_{r}$ are not necessarily distinct. Since $S / E(S) \cong$ $\left(H / \Gamma_{S}\right) /\left(N_{S} / \Gamma_{S}\right) \cong H / N_{S}$ is orientable, we get $\operatorname{sign}\left(\sigma\left(N_{S}\right)\right)=$ "+". Let $r$ be the number of elliptic elements in canonical generators of $N_{S}$. The orders of elliptic elements are divisors $(\neq 1)$ of $n$. We write them $d_{1}, \cdots, d_{r}$. Let $k$ be the number of period-cycles of $N_{S}$. Since there exists a symmetry on $S, N_{S}$ contains reflections. Hence it follows that $k \geq 1$. Since $N_{S} / \Gamma_{S} \cong E(S) \cong \mathbf{Z}_{2 n}$, there exists an epimorphism

$$
\eta: N_{S} \rightarrow \mathbf{Z}_{2 n}
$$

with $\operatorname{ker}(\eta)=\Gamma_{S}$. For every element $u$ of order 2 in $N_{S}$, we get $\eta(u)=n$. Thus, for $u, v$ in $N_{S}$ of order $2, \operatorname{ker}(\eta)$ contains $u v$. Since $\Gamma_{S}$ is a torsionfree group, $u v$ is not an element of finite order $>1$. Hence there are no periods in any period-cycles of $\sigma\left(N_{S}\right)$. Since $S / A(S) \cong H / N_{S}^{+}$and $S / A(S)$ has genus $m$, the genus of $\sigma\left(N_{S}^{+}\right)$is equal to $m$. By Corollary 2.2.5 in [4], we get the required forms of $\sigma\left(N_{S}\right)$ and $\sigma\left(N_{S}^{+}\right)$.

We shall show the assertion (a). First we assume $m=1$. The signature of $N_{S}^{+}$is of form

$$
\sigma\left(N_{S}^{+}\right)=\left(1 ;+;\left[d_{1}, d_{1}, \cdots, d_{r}, d_{r}\right] ;\{-\}\right) .
$$

The area of $\sigma\left(N_{S}^{+}\right)$is given by

$$
\mu\left(N_{S}^{+}\right)=2 \sum_{i=1}^{r}\left(1-\frac{1}{d_{i}}\right) .
$$


From $\mu\left(N_{S}^{+}\right)>0$ it follows that $r \geq 1$. All signatures with respect to maximal Fuchsian groups are known in Theorems 1,2 and 3 in [8]. From these known results it follows that in the case of $r=1, N_{S}^{+}$is not maximal, because $\sigma\left(N_{S}^{+}\right)=(1 ;+;[d, d] ;\{-\})$ for some divisor $d(\neq 1)$ of $n$. Hence, by Theorem 1 in [8], there exists a Fuchsian group $\Gamma^{\prime} \supset N_{S}^{+}$satisfying

$$
\left[\Gamma^{\prime}: N_{S}^{+}\right]=2 \text { and } \sigma\left(\Gamma^{\prime}\right)=(0 ;+;[2,2,2,2, d] ;\{-\})
$$

so that the generators of $\Gamma^{\prime}$ is represented by $y_{1}, \cdots, y_{5}$ with the relations

$$
y_{i}^{2}=I_{H}(1 \leq i \leq 4), y_{5}^{d}=I_{H} \text { and } y_{1} \cdots y_{5}=I_{H} .
$$

We see that $\Gamma^{\prime}$ includes $\Gamma_{S}$ as a normal subgroup by the following way.

Let $D_{n}$ be the dihedral group of order $2 n$, namely,

$$
\left.D_{n}=\langle a, b| a^{n}=b^{2}=(a b)^{2}=e \text { (unit element) }\right\rangle .
$$

Since $N_{S}^{+} / \Gamma_{S} \cong A(S) \cong \mathbf{Z}_{n} \cong\langle a\rangle$, there exists an epimorphism $\theta: N_{S}^{+} \rightarrow$ $\mathbf{Z}_{n}$ with $\operatorname{ker}(\theta)=\Gamma_{S}$. By $\left[\Gamma^{\prime}: N_{S}^{+}\right]=2$, we can write $\Gamma^{\prime}=N_{S}^{+} \cup N_{S}^{+} \gamma_{1}$ for some $\gamma_{1}$ in $\Gamma^{\prime}$. Therefore for each $y_{i}(1 \leq i \leq 4)$ there exists $y_{i}^{\prime}$ in $N_{S}^{+}$satisfying $y_{i}=y_{i}^{\prime} \gamma_{1}$. We note that $y_{5} \in N_{S}^{+}$. Then We can define an epimorphism $\varphi_{1}: \Gamma^{\prime} \rightarrow D_{n}$ satisfying

$$
\begin{aligned}
& \varphi_{1}\left(y_{i}\right)=\theta\left(y_{i}^{\prime}\right) b \text { for } 1 \leq i \leq 4, \\
& \varphi_{1}\left(y_{5}\right)=\theta\left(y_{5}\right) .
\end{aligned}
$$

Since $\operatorname{ker}\left(\varphi_{1}\right)=\Gamma_{S}, \Gamma_{S}$ is a normal subgroup of $\Gamma^{\prime}$. Hence $r \geq 2$ must hold because $N_{S}^{+}$is the normalizer of $\Gamma_{S}$ in $A(H)$.

Next we assume $\mathrm{m}=2$. The signature of $N_{S}^{+}$is of form

$$
\sigma\left(N_{S}^{+}\right)=\left(2 ;+;\left[d_{1}, d_{1}, \cdots, d_{r}, d_{r}\right] ;\{-\}\right) .
$$

By Theorems 1 and 2 in [8], $N_{S}^{+}$is not maximal in the case of $r=0$, because $\sigma\left(N_{S}^{+}\right)=(2 ;+;[-] ;\{-\})$. Then, by Theorem 1 in [8], there exists a Fuchsian group $\Gamma^{\prime \prime} \supset N_{S}^{+}$satisfying

$$
\left[\Gamma^{\prime \prime}: N_{S}^{+}\right]=2 \text { and } \sigma\left(\Gamma^{\prime \prime}\right)=(0 ;+;[2,2,2,2,2,2] ;\{-\}),
$$

so that the generators of $\Gamma^{\prime \prime}$ is represented by $z_{1}, \cdots, z_{6}$ with the relations $z_{i}^{2}=z_{1} \cdots z_{6}=I_{H}(1 \leq i \leq 6)$. Since $\left[\Gamma^{\prime \prime}: N_{S}^{+}\right]=2$, we can write $\Gamma^{\prime \prime}=N_{S}^{+} \cup N_{S}^{+} \gamma_{2}$ for some $\gamma_{2}$ in $\Gamma^{\prime \prime}$. Therefore for each $z_{i}$ there exists $z_{i}^{\prime}$ 
in $N_{S}^{+}$satisfying $z_{i}=z_{i}^{\prime} \gamma_{2}$. We can define an epimorphism $\varphi_{2}: \Gamma^{\prime \prime} \rightarrow D_{n}$ satisfying

$$
\varphi_{2}\left(z_{i}\right)=\theta\left(z_{i}^{\prime}\right) b \text { for } 1 \leq i \leq 6 .
$$

Since $\operatorname{ker}\left(\varphi_{2}\right)=\Gamma_{S}, \Gamma_{S}$ is a normal subgroup of $\Gamma^{\prime \prime}$. Hence $r \geq 1$ must hold because $N_{S}^{+}$is the normalizer of $\Gamma_{S}$ in $A(H)$. Thus the assertion (a) holds.

We put $g^{\prime}=(m+1-k) / 2$. Then the set of canonical generators of $N_{S}$ is represented by

$$
\left\{a_{i}, b_{i}\left(1 \leq i \leq g^{\prime}\right), x_{j}(1 \leq j \leq r), e_{l}, c_{l}=c_{l 0}(1 \leq l \leq k)\right\}
$$

with the relations

$$
x_{j}^{d_{j}}=I_{H}(1 \leq j \leq r), e_{l}^{-1} c_{l} e_{l} c_{l}=c_{l}^{2}=I_{H}(1 \leq l \leq k)
$$

and

$$
\prod_{j=1}^{r} x_{j} \prod_{l=1}^{k} e_{l} \prod_{i=1}^{g^{\prime}}\left[a_{i}, b_{i}\right]=I_{H}
$$

We put

$$
F=\left\{1 \leq l \leq k ; e_{l} \notin \Gamma_{S}\right\} \text { and } t=\# F .
$$

For each $l$ in $F$ we denote by $f_{l}$ the order of $\eta\left(e_{l}\right)$ in $\mathbf{Z}_{2 n}$, which is a divisor $(\neq 1)$ of $n$. Then $d_{1}, \cdots, d_{r}, f_{l}(l \in F)$ are required divisors. The equality (b) is shown by the Riemann-Hurwitz relation $\mu\left(\Gamma_{S}\right)=\left[N_{S}: \Gamma_{S}\right] \mu\left(N_{S}\right)$, namely,

$$
2 g-2=2 n\left(m-1+\sum_{i=1}^{r}\left(1-\frac{1}{d_{i}}\right)\right)
$$

The assertion (c) follows from the genus of $\sigma\left(N_{S}\right)$. The assertion (d) follows from $t=\# F$.

We shall show the assertion (e). Let $T$ be a symmetry on $S$. Since $\left\{I_{S}, T\right\}$ is a subgroup of $E(S) \cong N_{S} / \Gamma_{S}$, there exists a subgroup $\Gamma_{1}$ of $N_{S}$ satisfying $\Gamma_{1} / \Gamma_{S} \cong\left\{I_{S}, T\right\}$. Then $\Gamma_{1}=\eta^{-1}(\{0, n\})$. Since $H / \Gamma_{1} \cong$ $\left(H / \Gamma_{S}\right) /\left(\Gamma_{1} / \Gamma_{S}\right) \cong S /\langle T\rangle,|\operatorname{sp}(S)|$ is the number of period-cycles of $\sigma\left(\Gamma_{1}\right)$. Consequently we shall determine the signature of $\Gamma_{1}$. Since $\left[N_{S}: \Gamma_{1}\right]$ is odd, we get $\operatorname{sign}\left(\sigma\left(\Gamma_{1}\right)\right)=\operatorname{sign}\left(\sigma\left(N_{S}\right)\right)=$ "+" $([4 ;$ Theorem 2.1.2]). The order of $\Gamma_{1} x_{j}$ in $N_{S} / \Gamma_{1}$ is equal to that of $x_{j}$ in $N_{S}$. Hence there are no proper periods of $\sigma\left(\Gamma_{1}\right)\left(\left[4 ;\right.\right.$ Theorem 2.2.3]). Since $\sigma\left(N_{S}\right)$ does not have any period in all period-cycles, neither does $\sigma\left(\Gamma_{1}\right)$. For each $l$ in $F$, the order of $\Gamma_{1} e_{l}$ 
in $N_{S} / \Gamma_{1}$ is equal to $f_{l}$, so that by using Theorem 2.4.2 in [4] the number $k_{1}$ of period-cycles of $\sigma\left(\Gamma_{1}\right)$ is given by

$$
k_{1}=n(k-t)+\sum_{l \in F} \frac{n}{f_{l}}=n\left(k-\sum_{l \in F}\left(1-\frac{1}{f_{l}}\right)\right) .
$$

Hence the signature of $\Gamma_{1}$ is given by

$$
\sigma\left(\Gamma_{1}\right)=\left(g_{1} ;+;[-] ;\{\overbrace{(-), \cdots,(-)}^{k_{1}}\}\right),
$$

where $g_{1}=\left(g-k_{1}+1\right) / 2$. Since $\operatorname{sign}\left(\sigma\left(\Gamma_{1}\right)\right)=$ " + ", $S /\langle T\rangle$ is orientable, so that $\gamma=k_{1}$. Hence the assertion (e) holds.

If $r+t>0$, we put $M=1 . c . m .\left\{d_{1}, \cdots, d_{r}, f_{l}(l \in F)\right\}$. Then

$$
\left\langle\eta\left(x_{j}\right)(1 \leq j \leq r), \eta\left(e_{l}\right)(l \in F)\right\rangle \cong \mathbf{Z}_{M}
$$

The canonical relation $\prod_{j=1}^{r} x_{j} \prod_{l=1}^{k} e_{l} \prod_{i=1}^{g^{\prime}}\left[a_{i}, b_{i}\right]=I_{H}$ implies $\sum_{j=1}^{r} \eta\left(x_{j}\right)$ $+\sum_{l \in F} \eta\left(e_{l}\right)=0$ in $\mathbf{Z}_{2 n}$, so that we can take elements $\xi_{j}(1 \leq j \leq r), \varepsilon_{l}(l \in$ $F)$ in $\mathbf{Z}_{M}$ satisfying $o\left(\xi_{j}\right)=d_{j}, o\left(\varepsilon_{l}\right)=f_{l}$ and $\sum_{j=1}^{r} \xi_{j}+\sum_{l \in F} \varepsilon_{l}=0$. Therefore the assertion (f) follows from Lemma 1.

We shall show the assertion (g). If $k=m+1$ then the set of canonical generators of $N_{S}$ is represented by

$$
\left\{x_{j}(1 \leq j \leq r), e_{l}, c_{l}=c_{l 0}(1 \leq l \leq k)\right\}
$$

with the relations

$$
x_{j}^{d_{\jmath}}=I_{H}(1 \leq j \leq r), e_{l}^{-1} c_{l} e_{l} c_{l}=c_{l}^{2}=I_{H}(1 \leq l \leq k)
$$

and

$$
\prod_{j=1}^{r} x_{j} \prod_{l=1}^{k} e_{l}=I_{H}
$$

Since $\eta: N_{S} \rightarrow \mathbf{Z}_{2 n}$ is surjective, the image of $\eta$,

$$
\operatorname{Im}(\eta)=\left\langle\eta\left(x_{j}\right)(1 \leq j \leq r), \eta\left(e_{l}\right), \eta\left(c_{l}\right)(1 \leq l \leq k)\right\rangle
$$

contains elements of order $2 n$. Since $\eta\left(c_{l}\right)(1 \leq l \leq k)$ are elements of order 2 , it follows that l.c.m. $\left\{d_{1}, \cdots, d_{r}, f_{l}(l \in F)\right\}=n$. Thus the assertion ( $\mathrm{g}$ ) holds. Hence the proof of "only if" part is completely achieved. 
Conversely we shall show the "if" part. Let $n, m, g, \gamma, r, t, d_{1}, \cdots, d_{r+t}$ and $k$ be given numbers satisfying conditions $(\mathrm{a})$ to $(\mathrm{g})$. We put

$$
\sigma=\left(g^{\prime} ;+;\left[d_{1}, \cdots, d_{r}\right] ;\{\overbrace{(-), \cdots,(-)}^{k}\}\right),
$$

where $g^{\prime}=(m+1-k) / 2$. By $(\mathrm{c}), g^{\prime}$ is a non-negative integer. Since the area $\mu(\sigma)=m-1+\sum_{j=1}^{r}\left(1-1 / d_{j}\right)$ is positive by $(\mathrm{b})$, there exist NEC groups with signature $\sigma$. By Corollary 2.2.5 in [4] the canonical Fuchsian groups of such NEC groups have the signature

$$
\sigma^{+}=\left(m ;+;\left[d_{1}, d_{1}, \cdots, d_{r}, d_{r}\right] ;\{-\}\right) .
$$

From (a) it follows that

$$
\sigma^{+} \neq\left(1 ;+;\left[d_{i}, d_{i}\right] ;\{-\}\right) \text { and } \sigma^{+} \neq(2 ;+;[-] ;\{-\})
$$

Therefore, by Theorems 1 and 2 in [8], there exists a maximal Fuchsian group with signature $\sigma^{+}$, so that we have a maximal NEC group with signature $\sigma$. We denote it by $N$.

Let $\left\{a_{i}, b_{i}\left(1 \leq i \leq g^{\prime}\right), x_{j}(1 \leq j \leq r), e_{l}, c_{l}=c_{l 0}(1 \leq l \leq k)\right\}$ be the set of canonical generators of $N$ satisfying

$$
x_{j}^{d_{j}}=I_{H}(1 \leq j \leq r), e_{l}^{-1} c_{l} e_{l} c_{l}=c_{l}^{2}=I_{H} \quad(1 \leq l \leq k)
$$

and

$$
\prod_{j=1}^{r} x_{j} \prod_{l=1}^{k} e_{l} \prod_{i=1}^{g^{\prime}}\left[a_{i}, b_{i}\right]=I_{H}
$$

Assume $r+t>0$. By the condition (f) and Lemma 1 there exist $\xi_{j}$ in $\mathbf{Z}_{2 n}$ of order $d_{j}(1 \leq j \leq r+t)$ such that

$$
\sum_{j=1}^{r+t} \xi_{j}=0 \text { in } \mathbf{Z}_{2 n}
$$

We can define an epimorphism $\eta: N \rightarrow \mathbf{Z}_{2 n}$ satisfying

$$
\begin{aligned}
\eta\left(a_{1}\right) & =\eta\left(b_{1}\right)=2\left(\text { if } g^{\prime} \geq 1\right), \eta\left(a_{i}\right)=\eta\left(b_{i}\right)=0\left(2 \leq i \leq g^{\prime}\right), \\
\eta\left(x_{j}\right) & =\xi_{j}(1 \leq j \leq r, \text { if } r \neq 0), \\
\eta\left(c_{l}\right) & =n(1 \leq l \leq k), \\
\eta\left(e_{l}\right) & = \begin{cases}\xi_{r+l} & (1 \leq l \leq t, \text { if } t \neq 0), \\
0 & (t+1 \leq l \leq k) .\end{cases}
\end{aligned}
$$

Because $\eta$ is compatible with the relations in $N$, that is, 


$$
\begin{array}{cl}
x_{j}^{d_{j}}=I_{H} & \Rightarrow \eta\left(x_{j}^{d_{j}}\right)=d_{j} \xi_{j}=0(1 \leq j \leq r), \\
c_{l}^{2}=I_{H} & \Rightarrow \eta\left(c_{l}^{2}\right)=2 n(1 \leq l \leq k), \\
e_{l}^{-1} c_{l} e_{l} c_{l}=I_{H} & \Rightarrow \eta\left(e_{l}^{-1} c_{l} e_{l} c_{l}\right)=0(1 \leq l \leq k), \\
\prod_{j=1}^{r} x_{j} \prod_{l=1}^{k} e_{l} \prod_{i=1}^{g^{\prime}}\left[a_{i}, b_{i}\right]=I_{H} & \Rightarrow \eta\left(\prod_{j=1}^{r} x_{j} \prod_{l=1}^{k} e_{l} \prod_{i=1}^{g^{\prime}}\left[a_{i}, b_{i}\right]\right) \\
&
\end{array}
$$

We shall show that $\eta$ is surjective. Since $k \geq 1, \operatorname{Im}(\eta)$ contains $\eta\left(c_{1}\right)$ of order 2. Therefore it is sufficient to show that $\operatorname{Im}(\eta)$ contains an element of order $n$. If $g^{\prime} \geq 1$, then $\eta\left(a_{1}\right)$ and $\eta\left(b_{1}\right)$ are of order $n$ by the definition. If $g^{\prime}=0$, that is, $k=m+1$, then by $(\mathrm{g})$ there exist elements of order $n$ in $\operatorname{Im}(\eta)$. Thus $\operatorname{Im}(\eta)=\mathbf{Z}_{2 n}$.

We put

$$
\Gamma=\operatorname{ker}(\eta) \text { and } S=H / \Gamma .
$$

Then $\Gamma$ is an NEC group.

We shall show that $S$ is a required Riemann surface. By the definition of $\eta$, there exist no elliptic elements and orientation-reversing ones in $\Gamma$, so that the genus of $\sigma(\Gamma)$ is equal to $g$ by the Riemann-Hurwitz relation $\mu(\Gamma)=2 n \mu(N)$. Therefore $\Gamma$ is a Fuchsian group of signature $\sigma(\Gamma)=$ $(g ;+;[-] ;\{-\})$. Hence $S$ is a compact Riemann surface of genus $g$. Since $N$ is maximal and includes $\Gamma$ as a normal subgroup, $N$ is the normalizer of $\Gamma$ in $E(H)$. Therefore $E(S) \cong N / \Gamma \cong \mathbf{Z}_{2 n}$. We put $\Gamma_{2}=\eta^{-1}(\{0, n\})$. Since $\Gamma_{2} / \Gamma$ is a subgroup of order 2 in $N / \Gamma$, there exists a symmetry $T$ on $S$ such that

$$
\Gamma_{2} / \Gamma \cong\left\{I_{S}, T\right\} \subset E(S) .
$$

Thus $S$ is symmetric. From $[E(S): A(S)]=2$ it follows that $A(S) \cong \mathbf{Z}_{n}$. The genus of $\sigma\left(N^{+}\right)$is equal to $2 g^{\prime}+k-1=m$, so that the genus of $S / A(S) \cong H / N^{+}$is equal to $\mathrm{m}$. Thus $S$ is of type $(n, m)$. The orientability of $S / E(S)$ is derived from $S / E(S) \cong H / N$ and $\operatorname{sign}(\sigma(N))=$ "+".

We shall show $\operatorname{sp}(S)=\gamma$. Note the form of $\sigma\left(\Gamma_{1}\right)$ given in the "only if" part. Similarly we obtain

$$
\sigma\left(\Gamma_{2}\right)=\left(g_{2} ;+;[-] ;\{\overbrace{(-), \cdots,(-)}^{k_{2}}\}\right)
$$

and

$$
k_{2}=n(k-t)+\sum_{l=1}^{t} \frac{n}{d_{r+l}}=n\left(k-\sum_{l=1}^{t}\left(1-\frac{1}{d_{r+l}}\right)\right) \text {. }
$$


Since $S /\langle T\rangle \cong(H / \Gamma) /\left(\Gamma_{2} / \Gamma\right) \cong H / \Gamma_{2}$, we have $\operatorname{sp}(S)=k_{2}=\gamma$. Hence $S$ is a symmetric Riemann surface of type $(n, m)$ with $g(S)=g, \operatorname{sp}(S)=\gamma$, $E(S) \cong \mathrm{Z}_{2 n}$ and with the orientable quotient $S / E(S)$. The proof of "if" part is completely achieved.

CoRollary 2. If $\sum_{i=1}^{r}\left(1-1 / d_{i}\right)=\sum_{i=1}^{t}\left(1-1 / d_{r+i}\right)$ in the above theorem, then

$$
g(S)+k(S /\langle T\rangle)-1=\# A(S)(g(S / A(S))+k(S / E(S))-1),
$$

where $k(X)$ denotes the number of boundary components of $X$.

Proof. By (b) and (e), we get $g+\gamma-1=n(m+k-1)$.

\section{$\S 5$. Examples}

We shall show the simplest examples on our theorem.

EXAMPLE 1. In the case of $n=9$ and $m=1$, our theorem is reduced to the following:

There exists a symmetric Riemann surface $S$ of type $(9,1)$ with $g(S)=$ $g, \operatorname{sp}(S)=\gamma, E(S) \cong \mathbf{Z}_{18}$ and with the orientable quotient $S / E(S)$ if and only if there exist non-negative integers $r_{1}, r_{2}, t_{1}, t_{2}, \overbrace{3, \cdots, 3}^{r_{1}+t_{1}}$ and $\overbrace{9, \cdots, 9}^{r_{2}+t_{2}}$ such that :

(1) $r_{1}+r_{2} \geq 2$.

(2) $g=6 r_{1}+8 r_{2}+1$.

(3) $0 \leq t_{1}+t_{2} \leq 2$.

(4) $\gamma=2\left(9-3 t_{1}-4 t_{2}\right)$.

(5) We put $\mathbf{r}=\left(r_{1}, r_{2}\right)$ and $\mathbf{t}=\left(t_{1}, t_{2}\right)$, then

(5.1) $\mathbf{r}=(s, 0), s \geq 2 \Rightarrow \mathbf{t}=(0,2)$,

$(5.2) \mathbf{r}=(s, 1), s \geq 1 \Rightarrow \mathbf{t}=(0,1),(1,1),(0,2)$.

Then the possible genera $g$ and species $\gamma$ are listed as follows: 


\begin{tabular}{|c|c|c|c|c|c|c|c|c|c|c|c|}
\hline$g$ & 13 & 15 & 17 & 19 & 21 & 23 & 25 & 27 & 29 & 31 & $\cdots$ \\
\hline \multirow{5}{*}{$\gamma$} & 2 & 2 & 2 & 2 & 2 & 2 & 2 & 2 & 2 & 2 & \\
& & 4 & 4 & & 4 & 4 & 4 & 4 & 4 & 4 & \\
& & & 6 & & & 6 & 6 & & 6 & 6 & $\cdots$ \\
& & 10 & 10 & & 10 & 10 & 10 & 10 & 10 & 10 & \\
& & & 12 & & & 12 & 12 & & 12 & 12 & \\
& & & 18 & & & 18 & 18 & & 18 & 18 & \\
\hline
\end{tabular}

The following figure illustrates the relation of $g, \gamma$ and $\mathbf{r}$.

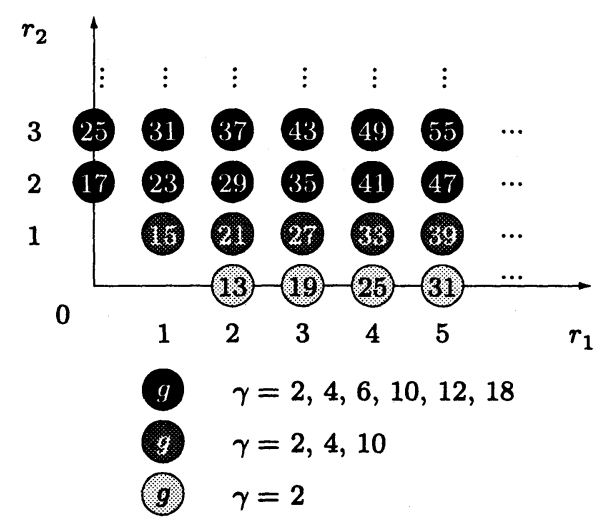

The following figure illustrates the relation of $g, \gamma$ and $\mathbf{t}$.

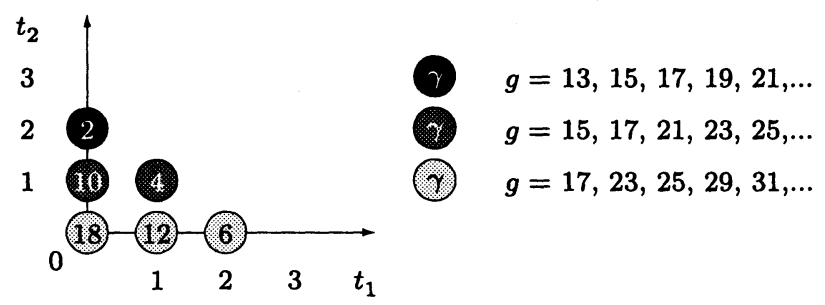

The possible $g$ and $\gamma$ satisfying the equality in Corollary 2 are the following

$$
\begin{array}{lll}
g=15 & \gamma=4 & (\mathbf{r}=\mathbf{t}=(1,1)) \\
g=17 & \gamma=2 & (\mathbf{r}=\mathbf{t}=(0,2))
\end{array}
$$

EXAMPLE 2. In the case of $n=15$ and $m=1$, our theorem is reduced to the following:

There exists a symmetric Riemann surface $S$ of type $(15,1)$ with $g(S)=$ $g, \operatorname{sp}(S)=\gamma, E(S) \cong \mathbf{Z}_{30}$ and with the orientable quotient $S / E(S)$ if and 
only if there exist non-negative integers $r_{1}, r_{2}, r_{3}, t_{1}, t_{2}, t_{3}, \overbrace{3, \cdots, 3}^{r_{1}+t_{1}}, \overbrace{5, \cdots, 5}^{r_{2}+t_{2}}$ and $\overbrace{15, \cdots, 15}^{r_{3}+t_{3}}$ such that:

(1) $r_{1}+r_{2}+r_{3} \geq 2$.

(2) $g=10 r_{1}+12 r_{2}+14 r_{3}+1$.

(3) $0 \leq t_{1}+t_{2}+t_{3} \leq 2$.

(4) $\gamma=2\left(15-5 t_{1}-6 t_{2}-7 t_{3}\right)$.

(5) We put $\mathbf{r}=\left(r_{1}, r_{2}, r_{3}\right)$ and $\mathbf{t}=\left(t_{1}, t_{2}, t_{3}\right)$, then

$$
\begin{aligned}
& \text { (5.1) } \mathbf{r}=(s, 0,0), s \geq 2 \Rightarrow \mathbf{t}=(0,2,0),(0,1,1),(0,0,2) \text {, } \\
& \text { (5.2) } \mathbf{r}=(0, s, 0), s \geq 2 \Rightarrow \mathbf{t}=(2,0,0),(1,0,1),(0,0,2) \text {, } \\
& \text { (5.3) } \mathbf{r}=(1,1,0) \quad \Rightarrow \mathbf{t}=(1,1,0),(1,0,1),(0,1,1) \text {, } \\
& (0,0,1),(0,0,2) \text {, } \\
& \text { (5.4) } \mathbf{r}=(1, s, 0), s \geq 2 \Rightarrow \mathbf{t} \neq(0, u, 0), u \geq 0 \text {, } \\
& \text { (5.5) } \mathbf{r}=(s, 1,0), s \geq 2 \Rightarrow \mathbf{t} \neq(u, 0,0), u \geq 0 \text {, } \\
& \text { (5.6) } \mathbf{r}=(s, 0,1), s \geq 1 \Rightarrow \mathbf{t} \neq(u, 0,0), u \geq 0 \text {, } \\
& \text { (5.7) } \mathbf{r}=(0, s, 1), s \geq 1 \Rightarrow \mathbf{t} \neq(0, u, 0), u \geq 0 \text {. }
\end{aligned}
$$

Then the possible genera $g$ and species $\gamma$ are listed as follows:

\begin{tabular}{|c|c|c|c|c|c|c|c|c|c|c|c|}
\hline$g$ & 21 & 23 & 25 & 27 & 29 & 31 & 33 & 35 & 37 & 39 & $\cdots$ \\
\hline & 2 & 2 & 2 & 2 & 2 & 2 & 2 & 2 & 2 & 2 & \\
& 4 & 4 & 4 & 4 & 4 & 4 & 4 & 4 & 4 & 4 & \\
& 6 & 6 & 6 & 6 & 6 & 6 & 6 & 6 & 6 & 6 & \\
& & 8 & 8 & 8 & 8 & & 8 & 8 & 8 & 8 & \\
$\gamma$ & & & 10 & 10 & 10 & & & 10 & 10 & 10 & $\ldots$ \\
& & 16 & 16 & 16 & 16 & & 16 & 16 & 16 & 16 & \\
& & & 18 & & 18 & & 18 & 18 & 18 & 18 & \\
& & & & 20 & 20 & & & 20 & 20 & 20 & \\
& & & & & 30 & & & & 30 & 30 & \\
\hline
\end{tabular}

The following figure illustrates the relation of $g, \gamma$, and $\mathbf{r}$. 


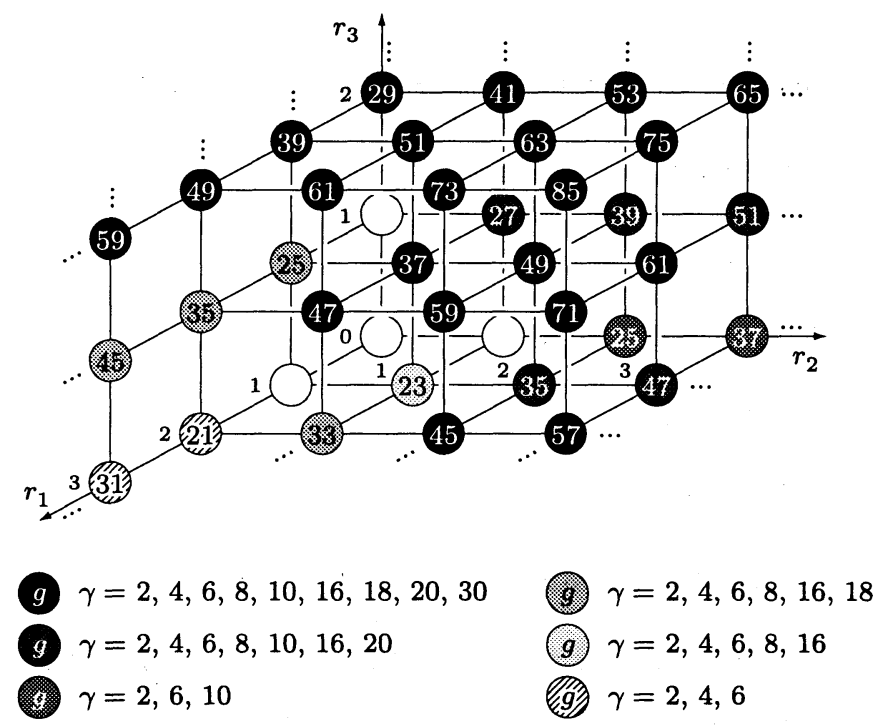

The following figure illustrates the relation of $g, \gamma$ and $\mathbf{t}$.

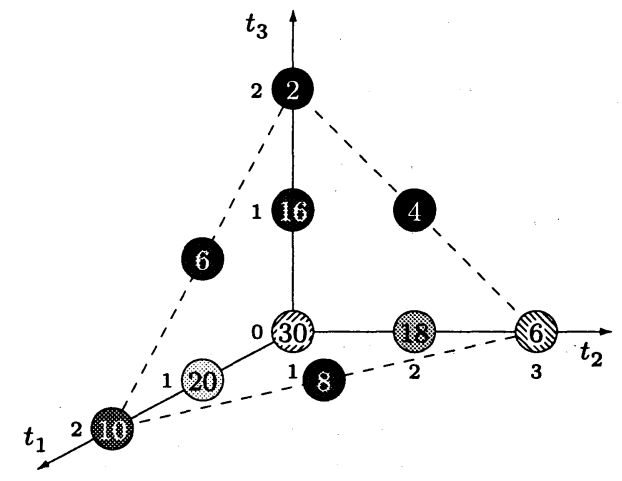
( $g=21,23,25,27,29,31, \ldots$
( $g=23,25,27,29,33,35, \ldots$
(2) $g=25,27,29,35,37,39, \ldots$
(9) $g=25,29,33,35,37,39, \ldots$
(7) $g=27,29,35,37,39, \ldots$
$g=29,37,39, \ldots$
$g=21,25,29,31, \ldots$

The possible $g$ and $\gamma$ satisfying the equality in Corollary 2 are the following

$$
\begin{array}{lll}
g=23 & \gamma=8 & (\mathbf{r}=\mathbf{t}=(1,1,0)) \\
g=25 & \gamma=6 & (\mathbf{r}=\mathbf{t}=(1,0,1)) \\
& & (\mathbf{r}=(1,0,1), \mathbf{t}=(0,2,0)) \\
& & (\mathbf{r}=(0,2,0), \mathbf{t}=(1,0,1)) \\
g=27 & \gamma=4 & (\mathbf{r}=\mathbf{t}=(0,1,1)) \\
g=29 & \gamma=2 & (\mathbf{r}=\mathbf{t}=(0,0,2))
\end{array}
$$




\section{REFERENCES}

[1] N. 1. Alling and N. Greenleaf, Foundations of the theory of Klein surfaces, Lecture Notes in Math., Vol. 219, Springer-Verlag, Berlin-New York, 1971.

[2] E. Bujalance, Normal subgroups of NEC groups, Math. Z., 178 (1981), 331-341.

[3] E. Bujalance, A. F. Costa and J. M. Gamboa, Real parts of complex algebraic curves, Lecture Notes in Math., Vol. 1420, Springer, Berlin, 1990, pp. 81-110.

[4] E. Bujalance, J. J. Etayo, J. M. Gamboa and G. Gromadzki, Automorphism groups of compact bordered Klein surfaces, Lecture Notes in Math., Vol. 1439, Springer-Verlag, Berlin, 1990.

[5] E. Bujalance and D. Singerman, The symmetry type of a Riemann surface, Proc. London Math. Soc., 51, No. 3 (1985), 501-519.

[6] A. M. Macbeath, The classification of non-Euclidean plane crystallographic groups, Canad. J. Math., 19 (1967), 1192-1205.

[7] C. Maclachlan, Maximal normal Fuchsian groups, Illinois J. Math., 15 (1971), 104-113.

[8] D. Singerman, Finitely maximal Fuchsian groups, J. London Math. Soc., 6, No. 2 (1972), 29-38.

[9] D. Singerman, On the structure of non-Euclidean crystallographic groups, Proc. Cambridge Philos. Soc., 76 (1974), 233-240.

[10] H. C. Wilkie, On non-Euclidean crystallographic groups, Math. Z., 91 (1966), 87-102.

Graduate School of Human Informatics

Nagoya University

Chikusa-ku, Nagoya 464-8601

Japan

nakamura@math.human.nagoya-u.ac.jp 